\title{
An unusual case of Escherichia coli meningitis and bacteremia in an elderly woman presenting with intractable low back pain
}

Andrea M. Lauffer, MD ${ }^{1}$, Mahmoud Shorman, $\mathrm{MD}^{2}$, Carl McComas, $\mathrm{MD}^{1}$

\section{Author Affiliations:}

1. St. Mary's Medical Center, Huntington, West Virginia

2. Joan C Edwards School of Medicine, Marshall University, Huntington, West Virginia

The authors have no financial disclosures to declare and no conflicts of interest to report.

Corresponding Author:

Andrea M. Lauffer, MD

St. Mary's Medical Center

Huntington, West Virginia

Email: lauffer1@marshall.edu 


\section{Abstract}

Introduction

We report an unusual case of E. coli meningitis in an elderly woman who presented to the emergency room with a chief complaint of intractable low back pain.

Case Description

A 67 year old woman presented to the emergency room with a chief complaint of intractable low back pain. After admission, the patient developed delirium. Blood cultures were drawn. Patient underwent a lumbar puncture which revealed purulent cerebrospinal fluid. Results of the cerebrospinal fluid and blood cultures revealed pan-sensitive E. coli.

Conclusion

In the geriatric population, delayed presentation of meningitis can occur for various reasons.

With the older adult population, existing co-morbidities and decline in immunologic function can mask symptoms. Clinicians caring for the elderly population need to have a high index of suspicion in the setting of subtle symptoms when it comes to diagnosing acute bacterial meningitis.

\section{Keywords}

bacteremia, meningitis, geriatric, E. Coli, clinical, cerebrospinal, cultures, delirium

\section{Introduction}

An elderly woman presented to the emergency room (ER) with intractable low back pain. She became delirious which prompted a further neurologic and infectious evaluation. This subsequently revealed bacterial invasion of the cerebrospinal fluid and blood with Escherichia coli. Prompt workup and treatment in the setting of a weak clinical presentation for meningitis and bacteremia resulted in full recovery and no neurologic sequelae for the patient.

In the geriatric population, delayed presentation of meningitis can occur for various reasons. Symptoms of bacterial meningitis tend to be more classic and pronounced in younger patients. Common symptoms of meningitis include nuchal rigidity, fever, altered mental status, and headache. But each of these symptoms is not individually specific or sensitive for the diagnosis of meningitis in the elderly. In the geriatric population, existing co-morbidities and decline in immunologic function can blunt the hallmark symptoms. Neck stiffness can be a result of ongoing arthritis. ${ }^{3}$ Fever may often be absent in older adults with an underlying infectious process. ${ }^{3}$ Altered mental status has a wide range of causes in the hospitalized elderly patient. Studies have demonstrated that first antibiotic administration time is delayed in older adults when compared to younger patients with acute bacterial meningitis. ${ }^{1}$ However, the hospital presentation time between younger and older adults with meningitis is nearly equal. ${ }^{1}$ This represents an unfortunate hindrance in the timing of diagnosis and treatment of bacterial meningitis in the elderly population. As a result, twenty percent of older adults die as a result of bacterial meningitis. ${ }^{3}$ 


\section{Case Description}

A 67 year old woman presented to the emergency room with a chief complaint of low back pain. Past medical history included hypertension, hypothyroidism, skin cancer requiring excision, and arthritis. Patient was unsure of the type of skin cancer she had. Her only treatment for the skin cancer was excision. Past surgical history included a tubal ligation. She denied history of smoking. She drank alcohol minimally and occasionally. Medications included losartan and synthroid. She had a very high functional status and was employed as a secretary at a local public sewer district.

Upon questioning by the ER staff, patient denied photophobia. She did complain of a headache and admitted to experiencing episodes of vomiting prior to coming to the emergency room. Her main complaint was severe low back pain starting in her lower back and radiating into both of her lower extremities. She also continued to have vomiting.

On exam by the emergency room physician, she was alert and afebrile. Temperature was 97.7 F, heart rate was 102 beats/minutes. Blood pressure was 135/92 mmHg. The patient did not have any meningeal signs or neck stiffness on exam. There was noted decrease flexion of her lumbar spine. Straight leg test of both lower extremities was negative. Patellar reflexes were intact bilaterally. No motor or sensory deficits noted. Motor strength was normal. Laboratory data obtained in the ER revealed a white blood cell count of 11,300. Platelets were 163,000. Urinalysis showed only trace leukocyte esterase. Complete metabolic profile revealed no gross abnormalities. Patient underwent extensive radiologic evaluation. Cat (CT) scan of her head without contrast showed no intracranial abnormality. CT scan of her lumbar spine without contrast showed degenerative changes. CT scan of the abdomen and pelvis without contrast showed fatty liver, a hiatal hernia, and pulmonary atelectasis versus pneumonia.

Hydromorphone was given to control the patient's back pain. She was also give ondansetron and prochlorperazine for her vomiting. Despite attempts to control her lower back pain in the ER, she continued to complain of severe lower back pain. She was admitted to the hospital floor for pain control.

On the second day of hospitalization, patient developed severe delirium and would not obey commands. On exam by the hospitalist, she was afebrile and hemodynamically stable. She did not have neck stiffness. Vomiting had resolved. Repeat white blood cell count was 12,300, and her platelets had dropped to 103,000. Repeat CT scan of the head without contrast showed no changes compared to the prior study.

A septic workup commenced. Two blood cultures were drawn. Lactic acid level was normal. Neurology was consulted. Patient underwent a lumbar puncture which revealed purulent cerebrospinal fluid (CSF). CSF analysis showed 1,883/microliter white blood cells, 724/microliter red blood cells, 81 neutrophils, 19 lymphocytes, $>460 \mathrm{mg} / \mathrm{dL}$ protein, and $<1$ $\mathrm{mg} / \mathrm{dL}$ glucose. Initial gram stain of the CSF showed gram negative bacillus. Eight hours after the blood cultures were drawn, they were positive for gram negative bacillus.

An infectious disease consult was placed. Prior to the evaluation by infectious disease, 
dexamethasone as well as acyclovir, vancomycin, ampicillin, and meropenem were started empirically by the hospitalist. The infectious disease physician narrowed antibiotic therapy to meropenem and one dose of gentamicin until identification and susceptibility profiles were available.

Eventually, identification and susceptibility profiles showed pan-sensitive E. coli as the cause of patient's bacteremia and bacterial meningitis. Her antibiotic therapy was narrowed to ceftriaxone. Neurologically, she returned to her baseline. Patient was discharged to the skilled nursing unit on hospital day thirteen where she completed rehabilitation therapy and a total of twenty-one days of intravenous ceftriaxone.

\section{Discussion}

The incidence of bacterial meningitis is 4 to 6 cases per 100,000 adults. ${ }^{7}$ However, the incidence of bacterial meningitis in adults over the age of 65 has declined. ${ }^{4}$ While $E$. coli is a common causative organism for meningitis in the neonatal patient population, it is not common in the older adult population. The three most common bacterial organisms in older patients with meningitis are Streptococcus pneumonia, Listeria monocytogenes, and Neisseria meningitidis. ${ }^{2}$ In eighty percent of adult cases, Streptococcus pneumoniae and Neisseria meningitidis are the responsible organisms. ${ }^{7}$ It is rare that community acquired meningitis is caused by a gram negative bacillus. ${ }^{5}$ When it does occur, community acquired $E$. coli meningitis is usually due to alcoholism, liver cirrhosis, neoplastic diseases, diabetes mellitus, and treatment with immunosuppressive agents. ${ }^{6}$

When considering the source of our patient's infection, all options were considered. CT scan of her abdomen revealed no abdominal cause of her infection. She was not up to date on her colon cancer screening. However, if the patient had an occult colon cancer or premalignant adenoma, Streptococcus bovis would have been the more likely bacterial culprit. ${ }^{8}$ Another possible infectious source would have been her sinuses, but CT scan of the head performed in the ER made note that her paranasal sinuses were clear. Given the patient's work exposure to gram negative organisms working at a local sewer district, this could have served as an introduction and exposure to the causative organism. When her delirium resolved, she was able to state to the medical team that sewer workers wearing their unclean field attire would often come into her office.

\section{Conclusion}

This case highlights what happens to many elderly patients who acquire bacterial meningitis. The absence of meningeal signs camouflaged the patient's true underlying diagnosis of bacterial meningitis. As this case demonstrates, classic meningeal signs are not always the presenting symptom of acute bacterial meningitis in an older adult.

Clinicians caring for the elderly population need to have a high index of suspicion in the setting of subtle symptoms when it comes to diagnosing acute bacterial meningitis. This will prevent delay in diagnosis and antibiotic administration time, and it will provide the patient a chance for a better clinical outcome. Severe low back pain was a clinical symptom of our patient's eventual 
development of meningitis. Her vomiting could have also been considered a symptom, but it resolved early in the hospital course and was deemed a nonspecific finding at presentation due to lack of meningeal signs and an unremarkable neurologic examination. Her development of acute delirium is what prompted further workup for meningitis. The challenging aspect of this case is that acute delirium in a hospital setting is not uncommon in an elderly patient. The differential diagnosis for the cause of delirium can be vast. However, as this case illustrates, meningitis should always be considered. Fortunately for our patient, a diagnosis of bacterial meningitis was made after her acute mental status change, and prompt initiation of antibiotic therapy helped the patient return to her normal baseline functional and neurologic status. If one had waited for meningeal signs to develop to pursue the diagnosis of bacterial meningitis for our patient, diagnosis and treatment would have been further delayed which would have been detrimental. Therefore, it is imperative that a physician master the clinical presentation of disease states in various age groups. Presenting signs and symptoms can be diverse and unique to a particular patient population. With regard to meningitis, special care and attention to the elderly population is essential in preventing delay in diagnosis and treatment. 


\section{References}

1. Domingo P, Pomar V, Benito ND, Coll P. The spectrum of acute bacterial meningitis in elderly patients. BMC Infectious Diseases. 2013;13(1).

2. Bacterial Meningitis [Internet]. Centers for Disease Control and Prevention. Centers for Disease Control and Prevention; 2016 [cited 20160ct16]. Available from: http://www.cdc.gov/meningitis/bacterial.html

3. Choi C. Bacterial Meningitis in Aging Adults. Clinical Infectious Diseases. 2001;33(8):1380-5.

4. Thigpen MC, Whitney CG, Messonnier NE, Zell ER, Lynfield R, Hadler JL, et al. Bacterial meningitis in the United States, 1998-2007. New England Journal of Medicine. 2011;364(21):2016-25.

5. Yoshino Y, Seo K, Koga I, Kitazawa T, Ota Y. A case of community-onset bacterial meningitis due to extended-spectrum beta-lactamase producing escherichia coli. J Med Cases Journal of Medical Cases. 2013;4(7):511-514.

6. Khan F, Abukhattab M, Anand D. Nosocomial escherichia coli meningitis in adults: report of four cases and literature review. Journal of Neurosciences in Rural Practice. 2013;4(3):349.

7. Beek DVD, Gans JD, Tunkel AR, Wijdicks EF. Community-acquired bacterial meningitis in adults. New England Journal of Medicine. 2006;354(1):44-53.

8. Boleij A, Schaeps RMJ, Tjalsma H. Association between streptococcus bovis and colon cancer. Journal of Clinical Microbiology. 2009;47(2):516. 